\title{
Plasticity in language cortex and white matter tracts after resection of dominant inferior parietal lobule arteriovenous malformations: a combined fMRI and DTI study
}

\author{
*Yuming Jiao, MD,,-4 Fuxin Lin, MD, ${ }^{5}$ Jun Wu, MD, ${ }^{1-4} \mathrm{Hao} \mathrm{Li,},{ }^{1-4}$ Weilun Fu, ${ }^{1-4}$ Ran Huo, MD, ${ }^{1-4}$ \\ Yong Cao, MD, ${ }^{1-4}$ Shuo Wang, MD, ${ }^{1-4}$ and Jizong Zhao, MD ${ }^{1-4}$
}

${ }^{1}$ Department of Neurosurgery, Beijing Tiantan Hospital, Capital Medical University, Beijing; ${ }^{2}$ China National Clinical Research Center for Neurological Diseases, Beijing; ${ }^{3}$ Center of Stroke, Beijing Institute for Brain Disorders, Beijing; ${ }^{4}$ Beijing Key Laboratory of Translational Medicine for Cerebrovascular Disease, Beijing; and ${ }^{5}$ Department of Neurosurgery, The First Affiliated Hospital of Fujian Medical University, Fuzhou, Fujian Province, People's Republic of China

OBJECTIVE The dominant inferior parietal lobe (IPL) contains cortical and subcortical structures that serve language processing. A high incidence of postoperative short-term aphasia and good potential for language reorganization have been observed. The authors' goal was to study the plasticity of the language cortex and language-related fibers in patients with brain arteriovenous malformations (BAVMs) located in the IPL.

METHODS A total of 6 patients who underwent microsurgical treatment of an IPL BAVM were prospectively recruited between September 2016 and May 2018. Blood oxygen level-dependent functional MRI (BOLD-fMRI) and diffusion tensor imaging (DTI) were performed within 1 week before and 6 months after microsurgery. Language-related white matter (WM) eloquent fiber tracts and their contralateral homologous fiber tracts were tracked. The Western Aphasia Battery was administered to assess language function. The authors determined the total number of fibers and mean fractional anisotropy (FA) indices for each individual tract. In addition, they calculated the laterality index (LI) between the activated language cortex voxels in the lesional and contralesional hemispheres and compared these indices between the preoperative and postoperative fMR and DT images.

RESULTS Of the 6 patients with IPL BAVMs, all experienced postoperative short-term language deficits, and $5(83.3 \%)$ recovered completely at 6 months after surgery. Five patients (83.3\%) had right homologous reorganization of BOLD signal activations in both Broca's and Wernicke's areas. More fibers were observed in the arcuate fasciculus (AF) in the lesional hemisphere than in the contralesional hemisphere (1905 vs 254 fibers, $p=0.035$ ). Six months after surgery, a significantly increased number of fibers was seen in the right hemispheric AF ( 249 fibers preoperatively vs 485 postoperatively, $p=0.026$ ). There were significantly more nerve fibers in the postoperative left inferior frontooccipital fasciculus (IFOF) (874 fibers preoperatively vs 1186 postoperatively, $p=0.010$ ). A statistically significant increase in right hemispheric dominance of Wernicke's area was observed. The overall functional LI showed functional lateralization of Wernicke's area in the right hemisphere $(\mathrm{LI} \leq-0.20)$ in all patients.

CONCLUSIONS The authors' findings provide evidence for the functional reorganization by recruiting the right hemispheric homologous region of Broca's and Wernicke's areas, right hemispheric AFs, and left hemispheric IFOFs following resection of IPL BAVMs.

Clinical trial registration no.: NCT02868008 (clinicaltrials.gov)

https://thejns.org/doi/abs/10.3171/2019.12.JNS191987

KEYWORDS dominant inferior parietal lobe; brain arteriovenous malformations; plasticity; language cortex; language-related fiber tracts; vascular disorders

ABBREVIATIONS AF = arcuate fasciculus; BAVM = brain arteriovenous malformation; BOLD = blood oxygen level-dependent; DSA = digital subtraction angiography; DTI = diffusion tensor imaging; FA = fractional anisotropy; $\mathrm{fMRI}=$ functional MRI; IFOF = inferior frontooccipital fasciculus; ILF = inferior longitudinal fasciculus; IPL = inferior parietal lobe; $\mathrm{LI}=$ laterality index; $\mathrm{ROI}=$ region of interest; SLF = superior longitudinal fasciculus; $\mathrm{TOF}=$ time of flight; $\mathrm{WM}=$ white matter.

SUBMITTED July 26, 2019. ACCEPTED December 10, 2019.

INCLUDE WHEN CITING Published online March 20, 2020; DOI: 10.3171/2019.12.JNS191987.

* Y.J. and F.L. contributed equally to this work. 
$\mathrm{T}$ HE dominant inferior parietal lobe (IPL), including the left supramarginal and angular gyri, sits at the temporoparietooccipital junction. ${ }^{5}$ Recent studies have identified it as an important hub for language processing, terming it Geschwind's area., ${ }^{5,26,27}$ This area is connected by large language-related fiber bundles to both Broca's and Wernicke's areas. It contains cortical and subcortical structures that serve language processing, such as the temporoparietal portion of the superior longitudinal fasciculus (SLF), SLF components II and III, the inferior frontooccipital fasciculus (IFOF), and the arcuate fasciculus (AF).,16,28 Previous studies indicated that lesions located in the IPL exhibited a higher rate of postoperative language deficits, especially of short-term language deficits, than Broca's and Wernicke's areas, ${ }^{12,19,21}$ which suggested that the IPL was significant in language processing.

The human brain has an intrinsic capacity to compensate for structural damage through reorganization of surviving networks. These processes are fundamental for regaining or preserving functions. The eloquent areas can be considered as nodes within a wide network. The entire functional network will self-reorganize by dynamic brain plasticity after the removal of these areas. ${ }^{9}$ According to a previous study, language function has a greater potential of postsurgical reorganization after injury to the IPL than after injury to Broca's and Wernicke's areas; more language deficits resulting from resection of lesions located in the IPL recovered to normal at the long-term clinic visit. ${ }^{12,20}$ However, longitudinal studies are required to reveal the structural plasticity occurring in the ipsilateral and contralateral brain regions and white matter (WM) following insult to this area.

In the clinic, blood oxygen level-dependent functional MRI (BOLD-fMRI) and diffusion tensor imaging (DTI) are valuable and noninvasive methods of mapping the eloquent areas and fiber tracts. They offer topographic maps of language function with very high sensitivity and acceptable specificity. ${ }^{4,18}$ In the present study, we used pre- and postsurgery BOLD-fMRI to study language cortex lateralization, and we applied DTI to study the plasticity of language-related fibers in patients with brain arteriovenous malformations (BAVMs) located in the dominant IPL. We provide evidence of reorganization of the language cortex and WM fasciculus by comparing the activated voxels of the language cortex, mean fractional anisotropy (FA), and number of fibers of the WM fiber tracts.

\section{Methods \\ Study Population}

In our database of a prospective BAVM clinical trial study (clinicaltrials.gov identifier NCT02868008), 6 patients who underwent microsurgical resection for an IPL BAVM were recruited between September 2016 and May 2018. This study was approved by the Institute Research Medical Ethics Committee of Beijing Tiantan Hospital Affiliated with Capital Medical University and adhered to good clinical practice guidelines according to the Declaration of Helsinki. Informed consent was documented in writing on an informed consent form approved by the IRB and signed by the patient or the patient's legally authorized representative.

\section{Neuroimaging}

MRI, CT, and digital subtraction angiography (DSA) scans were obtained in all patients. Functional MRI and DTI were performed using a 3.0T Siemens Trio MRI scanner within 1 week before surgery and 6 months after surgery. Imaging parameters for the sagittal T1-weighted anatomical images, BOLD-fMRI sequences, DT images, and time-of-flight (TOF) MRA images were reported in our previous work..$^{15}$

Patients underwent block-designed fMRI, and 2 lexical-semantic language paradigms were performed: picture naming and verb generation. For picture naming, the patient was asked to silently name each object as it appeared at the center of the patient's visual field. As a control, the patient was scheduled to passively view a picture of chopsticks. For verb generation, common concrete (written) nouns were displayed. The patient was asked to silently generate a verb associated with each noun. As a control, the patient passively viewed a pound sign (\#). Each paradigm contained a set of 40 tasks, which were divided equally into 8 performance periods; there were 5 tasks each interleaved with 8 control condition periods. The duration of a task was 3 seconds. All patients were trained with similar paradigms, but the order in which the tasks were presented during scanning was different from the order during training. All patients demonstrated the ability to perform the tasks. ${ }^{12}$

\section{Neuroimaging Data Analyses}

The iPlan 3.0 workstation (Brainlab) was used to manage the generated image sets. All image sets were automatically coregistered with each other and fused to the 3D T1-weighted images by an automatic rigid registration. A threshold of $p<0.001(T=6.5)$ was selected to determine the activated voxels. Two experienced neurosurgeons (J.W. and F.L.), on consensus, documented the anatomical locations of the activated cortex and peak activity point for each paradigm. The assessors were blinded to the patient outcomes. The bilateral long segment, the anterior and posterior segments of AF, the IFOF, the uncinate fasciculus, and the inferior longitudinal fasciculus (ILF) were studied as WM fiber tracts. Two regions of interest (ROIs) were used to track fiber tracts on axial slices. ${ }^{3,4} \mathrm{Fi}-$ ber tracking by fiber propagation stopped at an FA threshold of less than 0.20 and with a minimum fiber length of $70 \mathrm{~mm}$ was performed. Two neurosurgeons, on consensus, documented the anatomical locations of the ROIs and information of the WM fiber tracts.

\section{Surgery}

BAVMs were resected by an experienced senior physician (Y.C.) with the aid of a neuronavigation system. In all cases, intraoperative ultrasonography and indocyanine fluorescence angiography were used to discern the feeding arteries and the margin of BAVMs. Radical obliteration was validated by DSA 5 days after surgery. 
TABLE 1. Summary of treated patients with IPL BAVMs

\begin{tabular}{ccccccccccc}
\hline $\begin{array}{c}\text { Patient } \\
\text { No. }\end{array}$ & $\begin{array}{c}\text { Age (yrs), } \\
\text { Sex }\end{array}$ & $\begin{array}{c}\text { Spetzler-Martin } \\
\text { Grade }\end{array}$ & $\begin{array}{c}\text { Size } \\
(\mathrm{mm})\end{array}$ & $\begin{array}{c}\text { Preferred } \\
\text { Hand }\end{array}$ & Onset & $\begin{array}{c}\text { Preop } \\
\text { LD }\end{array}$ & $\begin{array}{c}\text { Type of Aphasia } \\
\text { 1 Wk Postop }\end{array}$ & $\begin{array}{c}\text { Lt Fibers } \\
\text { Injured }\end{array}$ & $\begin{array}{c}\text { LD at } 6 \text { Mos } \\
\text { Postop }\end{array}$ \\
\hline 1 & $38, \mathrm{M}$ & II & 46.8 & $\mathrm{Rt}$ & Seizure & No & Wernicke's & AF & No \\
\hline 2 & $20, \mathrm{M}$ & III & 33.9 & $\mathrm{Rt}$ & Seizure & No & Isolation & AF, IFOF & Yes \\
\hline 3 & $19, \mathrm{~F}$ & III & 47 & $\mathrm{Rt}$ & Seizure & No & Conduction & AF & No \\
\hline 4 & $21, \mathrm{M}$ & III & 30.7 & $\mathrm{Rt}$ & Hemorrhage & Yes & Anomic & AF & No \\
\hline 5 & 14, F & IV & 67.1 & Rt & Headache & No & Conduction & AF, IFOF, ILF & No \\
\hline 6 & $33, \mathrm{M}$ & III & 61.6 & Rt & Headache & No & Anomic & AF & No \\
\hline
\end{tabular}

$\mathrm{LD}=$ language deficit.

\section{Language Evaluation}

Left-handed patients were excluded based on the Edinburgh Handedness Inventory before surgery. The Western Aphasia Battery (WAB) was used to test the presence and type of language deficit. Patients who had any hearing disturbance or motor deficit due to pyramidal tract disturbance were also excluded, because both could affect the WAB results. Language function assessments were performed by the same experienced neurosurgeon (H.L.) before surgery and at 7 days and 6 months after surgery.

\section{Statistical Analyses}

Statistical analyses were performed using the statistical package IBM SPSS (version 20.0.0, IBM Corp.). The number of fibers and mean FA values for each individual WM fiber tract in different hemispheres and patients were documented. This parameter was defined as a quantitative measure of connectivity between eloquent cortices as determined by the ROIs. Furthermore, we calculated the laterality index (LI) based on the activated language cortex voxels in lesional and contralesional hemispheres and compared these values between the preoperative and postoperative fMR images. LIs for the control group were calculated using the following equation: (value left $_{-}$ value $\left._{\text {right }}\right) /\left(\right.$ value $_{\text {left }}+$ value $\left._{\text {right }}\right)$. The values range from 1 to -1 . Negative values indicate fewer activated voxels in the affected hemisphere, a value of 0 means an equal number of voxels in both hemispheres, and positive values indicate fewer voxels in the dominant hemisphere than in the contralesional hemisphere. An LI of $\leq-0.2$ was defined as right-sided lateralization of BOLD signals, and an LI of $\geq$ 0.2 was considered left-sided lateralization; hemispheric preference was not clear when the LI was between -0.2 and 0.2. ${ }^{14}$ One-way ANOVA was undertaken to test the group differences in mean FA and number of fibers between preoperative and postoperative images; $p<0.05$ was considered statistically significant.

\section{Results}

\section{Participants and Clinical Outcomes}

Between September 2016 and May 2018, 6 patients (4 males and 2 females; mean age 24.2 years, range 14-38 years) with IPL BAVMs were recruited at the Beijing Tiantan Hospital. All patients were right-handed native Chinese speakers and reported no history of a hearing disorder. The initial presentation was seizure in 3 patients, headache in 2 patients, and hemorrhagic onset in 1 patient. The hemorrhagic patient experienced preoperative language deficits. Radical resection was achieved in all patients according to postoperative DSA findings. All patients (100\%) experienced postoperative short-term language deficits. At the 6-month follow-up, 5 patients (83.3\%) with short-term language deficits recovered completely (Table 1), and 1 patient (16.7\%) recovered partially. Deaths and rehemorrhages did not occur during the follow-up.

\section{Preoperative MRI Analysis}

The mean number of fibers for each investigated set of fibers on preoperative images is presented in Table 2. For the left hemispheric AF, the anterior and posterior segments could be tracked out in all patients except for patient 4 . However, the long segment could only be tracked out in 3 patients (patients 2, 5, and 6). More fibers were observed in the AF in the lesional hemisphere than in the contralesional hemisphere (1905 vs $254, \mathrm{p}=0.035$; mean $\mathrm{LI}=0.35$ ) in patients with left AFs. All other fiber tracts could be tracked out. Significantly more fibers were observed in the ILF in the lesional hemisphere than in the contralesional hemisphere (4112 vs 2898, $\mathrm{p}=0.011$; mean $\mathrm{LI}=0.16)$. No significant difference was observed in the laterality of the other language-related fibers.

For all individuals, in addition to the classic predefined areas in the left hemisphere, language-related activation in Broca's and Wernicke's areas was also observed in their homologous area of the right hemisphere in 5 patients (83.3\%). The overall functional LI showed left-sided lateralization of Broca's and Wernicke's areas ( $L I \geq 0.20)$ in all patients. There was a significant trend of Broca's area showing stronger left-sided laterality than Wernicke's area (0.803 vs $0.169, \mathrm{p}=0.080$ ) (Table 3 ).

\section{Postoperative DTI Analysis}

We analyzed the plasticity of language-related WM fiber tracts using the DT images acquired 6 months after surgery. Patients had greater plasticity of the fibers on postoperative images than on preoperative images. The left AF in all 6 patients was injured after surgery. Only posterior segments of the left AF in 3 patients (patients 1 , 2 , and 6) could be tracked out. Patients had a significantly increased number of fibers in the right AF (249 fibers 
TABLE 2. Mean DTI indices for each white matter tract pre- and postoperatively, and results of between-group comparisons

\begin{tabular}{|c|c|c|c|c|c|c|}
\hline & \multicolumn{3}{|c|}{ No. of Fibers } & \multicolumn{3}{|c|}{ FA } \\
\hline & \multicolumn{2}{|c|}{ Mean (SD) } & \multirow[b]{2}{*}{$p$ Value } & \multicolumn{2}{|c|}{ Mean (SD) } & \multirow[b]{2}{*}{$p$ Value } \\
\hline & Preop & Postop & & Preop & Postop & \\
\hline Rt AF & $249(170)$ & $485(170)$ & 0.026 & $0.402(0.020)$ & $0.415(0.012)$ & 0.082 \\
\hline Rt UF & $312(349)$ & $585(1714)$ & 0.115 & $0.385(0.023)$ & $0.397(0.029)$ & 0.201 \\
\hline Rt ILF & $2898(2794)$ & $3638(2848)$ & 0.630 & $0.430(0.019)$ & $0.417(0.010)$ & 0.207 \\
\hline Rt IFOF & 1870 (1238) & $1705(1028)$ & 0.577 & $0.437(0.016)$ & $0.430(0.017)$ & 0.235 \\
\hline \multicolumn{7}{|l|}{$\mathrm{Lt}_{\mathrm{AF}}^{*}$} \\
\hline Lt UF & $698(576)$ & $523(864)$ & 0.536 & $0.395(0.027)$ & $0.420(0.031)$ & 0.125 \\
\hline Lt ILF & 4112 (1922) & 2406 (1759) & 0.031 & $0.438(0.015)$ & $0.398(0.036)$ & 0.034 \\
\hline Lt IFOF & $874(592)$ & $1186(523)$ & 0.010 & $0.438(0.026)$ & $0.473(0.010)$ & 0.060 \\
\hline
\end{tabular}

UF = uncinate fasciculus

Boldface type indicates statistical significance.

* Left AF values were not compared because the postoperative AF was injured in all patients.

preoperatively vs 485 postoperatively, $\mathrm{p}=0.026)$. The left IFOFs in 2 patients (patients 2 and 6 ) were injured after surgery and could not be tracked out. The remaining 4 patients had significantly more fibers in the left IFOF $(874$ fibers preoperatively vs 1186 postoperatively, $\mathrm{p}=0.010$ ) (Fig. 1). For the ILF, the left ILF in 1 patient (patient 5) was injured after surgery and could not be tracked out. The remaining 5 patients had significantly fewer fibers in the left ILFs (4112 fibers preoperatively vs 2406 postoperatively, $p=0.031$ ). No significant differences were observed between the pre- and postoperative uncinate fasciculi.

\section{Functional Hemispheric Language Lateralization (BOLD-fMRI)}

We analyzed the language cortex activation using BOLD-fMR images acquired at 6 months after surgery. Patients had a statistically significant increase in right hemispheric activation of Broca's area. The pre- and postoperative LIs of Broca's area were 0.843 and 0.379 , respectively ( $p=0.011$; Figs. 2 and 3 and Table 3). Meanwhile, the patients had a statistically significant increase in right-sided laterality of Wernicke's area. The pre- and

TABLE 3. LI index of language area activation according to fMRI

\begin{tabular}{|c|c|c|c|c|c|c|}
\hline \multirow{2}{*}{$\begin{array}{c}\text { Patient } \\
\text { No. }\end{array}$} & \multicolumn{3}{|c|}{ Broca's Area } & \multicolumn{3}{|c|}{ Wernicke's Area } \\
\hline & Preop LI & Postop LI & $p$ Value & Preop LI & Postop LI & $p$ Value \\
\hline 1 & 0.643 & 0.439 & \multirow{8}{*}{0.011} & 0.178 & -0.590 & \multirow{8}{*}{0.021} \\
\hline 2 & 0.970 & 0.589 & & 0.043 & -1.000 & \\
\hline 3 & 0.904 & 0.635 & & -0.315 & -1.000 & \\
\hline 4 & 0.349 & -0.383 & & 0.250 & -1.000 & \\
\hline 5 & 1.000 & 0.809 & & 1.000 & -1.000 & \\
\hline 6 & 0.953 & 0.185 & & -0.143 & -1.000 & \\
\hline Mean & 0.803 & 0.379 & & 0.169 & -0.932 & \\
\hline SD & 0.403 & 0.428 & & 0.687 & 0.168 & \\
\hline
\end{tabular}

Boldface type indicates statistical significance. postoperative LIs of Wernicke's area were 0.169 and -0.932 , respectively $(p=0.021)$. The overall functional LI showed right-sided laterality of Wernicke's area (LI $\leq$ $-0.20)$ in all patients.

\section{Discussion}

The language-dominant IPL contains critical cortical and subcortical brain structures for language function. ${ }^{24}$ Few reports have characterized language area plasticity after surgical removal of BAVMs from this brain region. In this study, we report the reorganization patterns throughout a consecutive series specifically dedicated to the resection of IPL BAVMs. The current study provides evidence for functional reorganization by recruiting the right hemispheric homologous region of Broca's and Wernicke's areas, right hemispheric AF, and left hemispheric IFOF following resection of IPL BAVMs.

In our study, a high incidence of right hemispheric activation was noted in the homologous area of both Broca's and Wernicke's areas before surgery. Previous studies demonstrated right lateralization of BOLD signal activations in BAVMs located in classic language cortex, and there was a significant difference between BOLD signal activation in patients with a BAVM in Broca's area and Wernicke's area. A nidus involving Broca's area or Wernicke's area mainly results in right-sided lateralization of the area ${ }^{6,25}$ According to our data, right-hemispheric activation was noticed in the homologous area of both Broca's and Wernicke's areas. We speculate that it resulted from the important role of the IPL in the language network. A lesion in the IPL might interfere with the interplay between Broca's and Wernicke's areas and lead to reorganization of both areas. Different from lesions in Broca's and Wernicke's areas, the dominant language cortex activation remained in the left hemisphere in the present study. This nondominant "mirror phenomenon" suggests that the right hemispheric homologous language cortex may play supplementary roles when the corresponding dominant language cortex is impaired. 


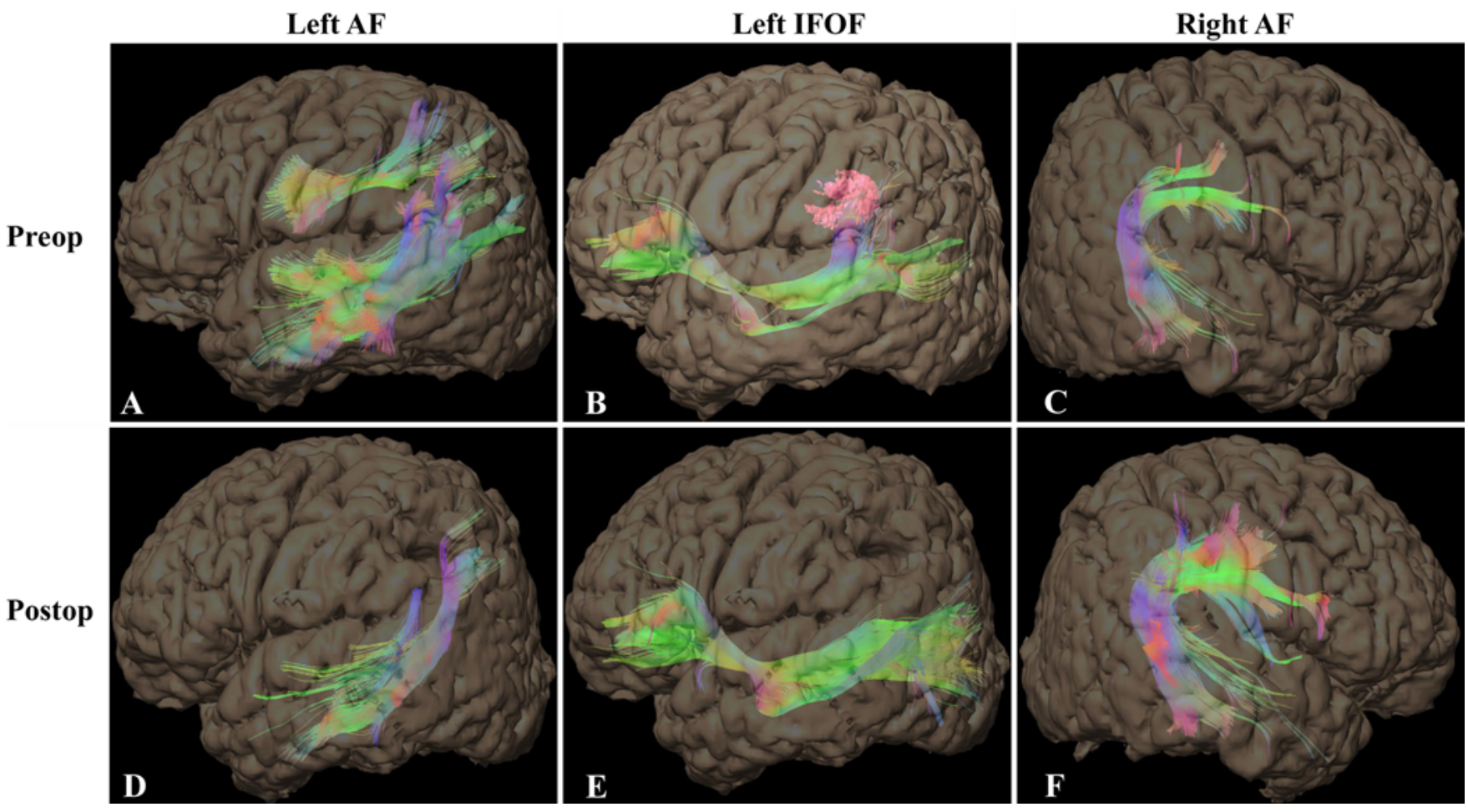

FIG. 1. DTI tractography images showing a comparison between pre- and postoperative language-related fiber tracts. Six months after surgery, the left $A F$ was ruptured, and only part of the posterior segment could be tracked out ( $A$ and $D)$. The fiber numbers of the left IFOF ( $B$ and $E$ ) and right $A F(C$ and $F$ ) increased significantly compared with the preoperative status. The language function of this patient recovered to normal 6 months after surgery. Figure is available in color online only.
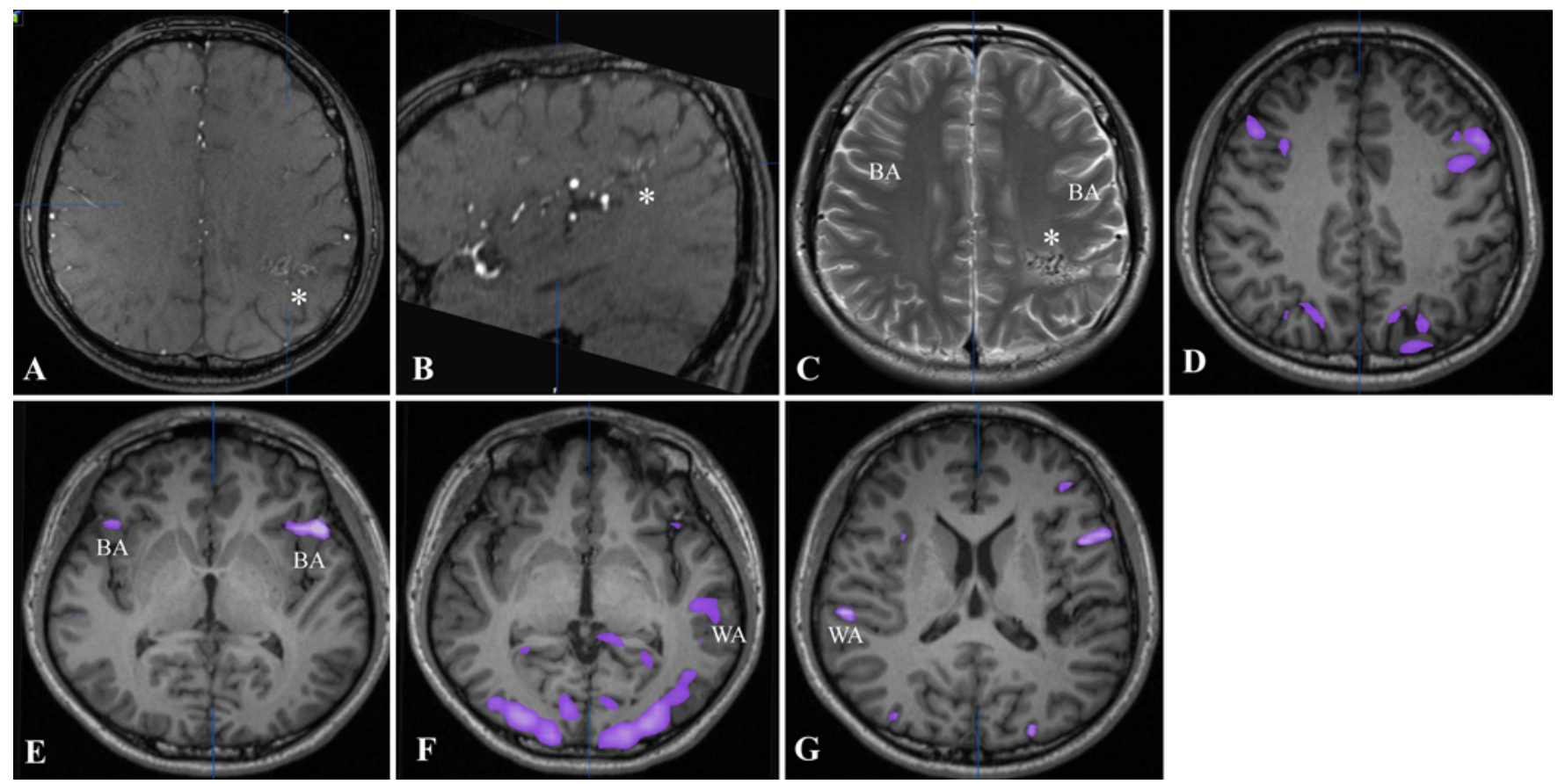

FIG. 2. Patient 1. MR images obtained in an IPL BAVM patient. Axial (A) and sagittal (B) TOF MR images revealing an AVM (asterisk) located in the IPL. Axial T2-weighted image (C) showing the flow voids of the AVM nidus (asterisk). Functional axial MR images showing right-sided reorganization of BOLD signal activation of both Broca's (D and E) and Wernicke's (F and $G$ ) areas (BA and WA, respectively). The dominant activation is located in the left hemisphere. Figure is available in color online only. 

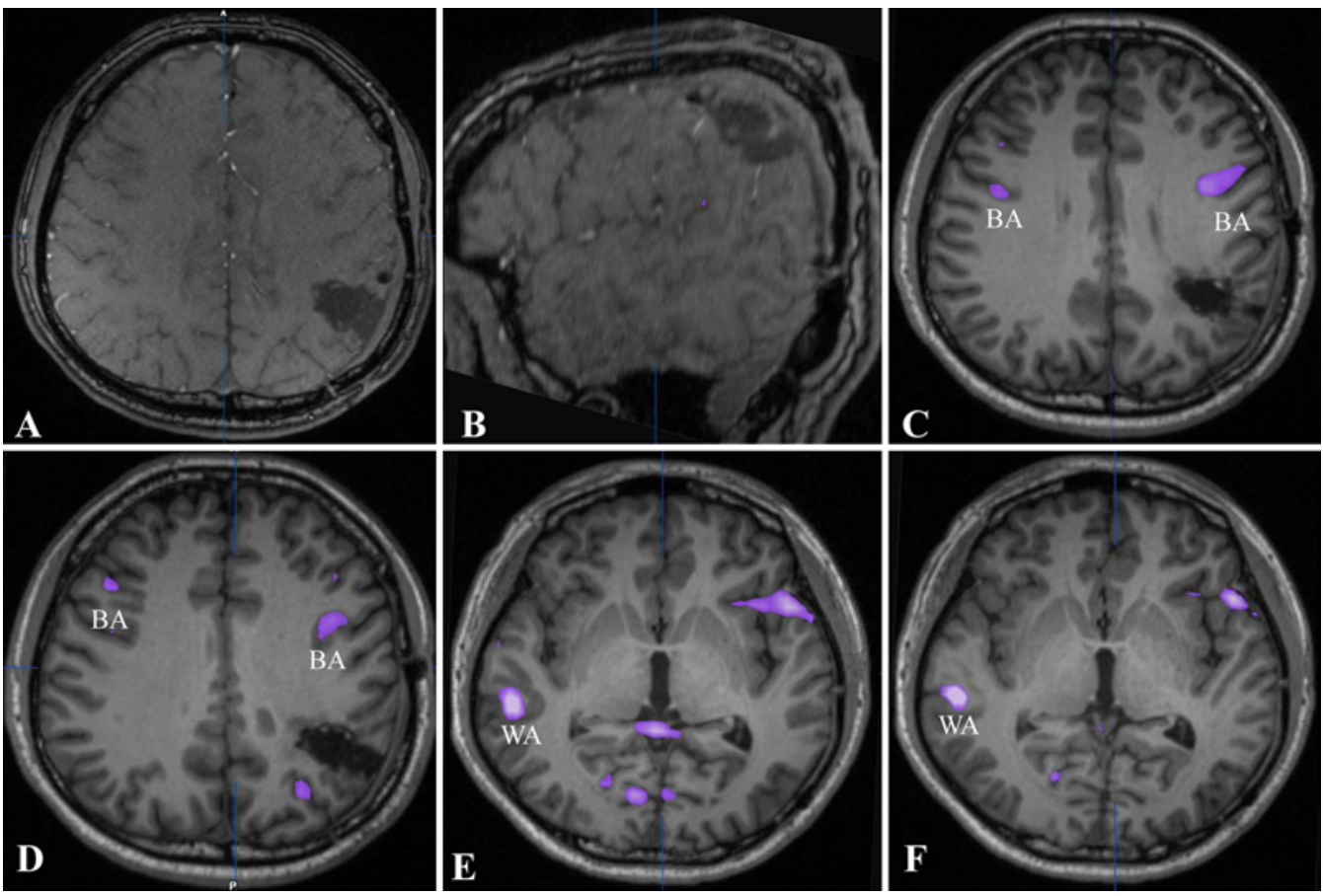

FIG. 3. Patient 1. Postoperative axial (A) and sagittal (B) TOF images showing the radical obliteration of the AVM and interruption of the IPL. Axial fMR images showing right-sided reorganization of BOLD signal activation of both Broca's (C and D) and Wernicke's ( $E$ and $F$ ) areas. The dominant activation of Broca's area remains in the left hemisphere, whereas the dominant activation of Wernicke's area shifted to the right. Figure is available in color online only.

In this study, 4 different types of aphasia were caused by injury to the IPL after surgery. We postulated that this variety was caused by the disconnection of complex interplay through this territory. The language-dominant IPL contains critical cortical and subcortical brain structures for language processing, including the cortical supramarginal and angular gyri, as well as subcortical WM fiber bundles such as the SLF, AF, and IFOF. According to previous studies, different segments of the dominant language fibers were differentially involved in language production and comprehension..$^{10}$ Lesion symptom mapping studies have shown the significance of the AF for both sustaining speech production and its temporal portion in supporting semantic integration. ${ }^{13} \mathrm{FA}$ indices for the middle and posterior segments of both the ILF and IFOF were correlated strongly with language comprehension. ${ }^{2}$ The various types of aphasia after surgery might be due to an injury to different segments of language-related fiber tracts. Further study is needed to confirm the relationship of aphasia type and the different injury patterns.

Our results showing an important role of the right AF in language plasticity are in line with a series of previous studies. In the present study, all the left AFs were ruptured after surgery, and reorganization of right AFs was observed. According to a previous report, there is reorganization of the right AFs following left AF resection in children with intractable epilepsy. ${ }^{7}$ Moreover, we observed an increase in the number of fibers and FA in the IFOF. The IFOF is a ventral associative fiber tract that connects the frontal cortex with the occipital and parietal lobes. Recent anatomical studies have ascertained the involvement of the left IFOF in the ventral semantic stream of language. ${ }^{1}$ According to previous revelations, IPL is the connector hub of the human semantic processing modules. ${ }^{8,26}$ It was reasonable to speculate that the unruptured left IFOF plays an important role in compensating for the semantic language functions after IPL injury. This result suggests that preservation of the IFOF may be of significance in the resection of lesions located in the IPL. Further studies are necessary to prove this hypothesis.

Six months after surgery, IPL BAVM patients demonstrated a right homologous reorganization of Broca's and Wernicke's areas during the language task. These results are in agreement with previous literature, which have shown right-sided language lateralization in patients with a left-sided epileptic focus who underwent resection for temporal lobe epilepsy. Nevertheless, Wernicke's area was significantly more right lateralized in the homologous areas than Broca's area in this study. We postulate that it was because Wernicke's area and the IPL may have more interplay in language processing. In a previous study, Thivard et al. described that productive and perceptive functions were differently reorganized by the pathological process. ${ }^{22}$ In patients with left temporal lobe epilepsy, they demonstrated a strong right lateralization of Wernicke's area during the perceptive language task and a strong right lateralization of Broca's area during the productive language task. It is now accepted that the brain sustains language processing via complex, sophisticated networks and that the traditional viewpoint that language is "located" in specific brain areas such as Broca's and Wernicke's areas is oversimplified..$^{23}$ According to previous studies, IPL is also 
involved in speech perceptive functions. ${ }^{11,17}$ Therefore, it is reasonable to assume that some of the speech perceptive function-related areas translocated to the contralateral hemisphere as a compensation of the injury to the IPL. Further study needs to be done to confirm the function of the reorganized language cortex.

\section{Limitations}

Several limitations of the study should be addressed. First, the sample size of the patients is too small to study the correlation between the neuroimaging plasticity and the recovery of different language components. Additional studies with a larger sample size with different symptom subgroups are needed in our future work. Second, this was an fMRI and DTI study, and confirmatory tests (electrocortical stimulation mapping and Wada testing) were not conducted. Further confirmation with a larger sample of subjects undergoing other confirmatory tests is needed to corroborate our conclusions.

\section{Conclusions}

Taken together, our findings provide evidence for functional reorganization by recruiting the right hemispheric homologous region of Broca's and Wernicke's areas, right hemispheric AFs, and left hemispheric IFOFs after resection of IPL BAVMs.

\section{Acknowledgments}

This study was supported by the National Natural Science Foundation of China (grant no. 81901175, principal investigator: Yuming Jiao), the "National key research and development program of China during the 13th Five-Year Plan Period" (grant no. 2016YFC1301803, principal investigator: Professor Yong Cao; and grant no. 2016YFC1301801, principal investigator: Professor Shuo Wang), Fujian Provincial Health and Family Planning Scientific Research Personnel Training Project (grant no. 2018-ZQN-43, principal investigator: Fuxin Lin), a grant from the Health and Family Planning Commission of Fujian Province, and the "Key Project of Beijing Municipal Science \& Technology Commission" (grant no. D161100003816006, principal investigator: Professor Shuo Wang; and grant no. D161100003816005, principal investigator: Professor Jizong Zhao).

\section{References}

1. Almairac F, Herbet G, Moritz-Gasser S, de Champfleur NM, Duffau H: The left inferior fronto-occipital fasciculus subserves language semantics: a multilevel lesion study. Brain Struct Funct 220:1983-1995, 2015

2. Bajada CJ, Lambon Ralph MA, Cloutman LL: Transport for language south of the Sylvian fissure: The routes and history of the main tracts and stations in the ventral language network. Cortex 69:141-151, 2015

3. Catani M, Jones DK, ffytche DH: Perisylvian language networks of the human brain. Ann Neurol 57:8-16, 2005

4. Catani M, Thiebaut de Schotten M: A diffusion tensor imaging tractography atlas for virtual in vivo dissections. Cortex 44:1105-1132, 2008

5. De Benedictis A, Duffau H, Paradiso B, Grandi E, Balbi S, Granieri E, et al: Anatomo-functional study of the temporoparieto-occipital region: dissection, tractographic and brain mapping evidence from a neurosurgical perspective. J Anat 225:132-151, 2014

6. Deng X, Xu L, Zhang Y, Wang B, Wang S, Zhao Y, et al: Dif- ference of language cortex reorganization between cerebral arteriovenous malformations, cavernous malformations, and gliomas: a functional MRI study. Neurosurg Rev 39:241249,2016

7. Goradia D, Chugani HT, Govindan RM, Behen M, Juhász C, Sood S: Reorganization of the right arcuate fasciculus following left arcuate fasciculus resection in children with intractable epilepsy. J Child Neurol 26:1246-1251, 2011

8. Han Z, Ma Y, Gong G, He Y, Caramazza A, Bi Y: White matter structural connectivity underlying semantic processing: evidence from brain damaged patients. Brain 136:29522965,2013

9. Ius T, Angelini E, Thiebaut de Schotten M, Mandonnet E, Duffau H: Evidence for potentials and limitations of brain plasticity using an atlas of functional resectability of WHO grade II gliomas: towards a "minimal common brain". Neuroimage 56:992-1000, 2011

10. Ivanova MV, Isaev DY, Dragoy OV, Akinina YS, Petrushevskiy AG, Fedina ON, et al: Diffusion-tensor imaging of major white matter tracts and their role in language processing in aphasia. Cortex 85:165-181, 2016

11. Jäncke L, Kleinschmidt A, Mirzazade S, Shah NJ, Freund HJ: The role of the inferior parietal cortex in linking the tactile perception and manual construction of object shapes. Cereb Cortex 11:114-121, 2001

12. Jiao Y, Lin F, Wu J, Li H, Chen X, Li Z, et al: Brain arteriovenous malformations located in language area: surgical outcomes and risk factors for postoperative language deficits. World Neurosurg 105:478-491, 2017

13. Kümmerer D, Hartwigsen G, Kellmeyer P, Glauche V, Mader I, Klöppel S, et al: Damage to ventral and dorsal language pathways in acute aphasia. Brain 136:619-629, 2013

14. Lee DJ, Pouratian N, Bookheimer SY, Martin NA: Factors predicting language lateralization in patients with perisylvian vascular malformations. Clinical article. J Neurosurg 113:723-730, 2010

15. Lin F, Zhao B, Wu J, Wang L, Jin Z, Cao Y, et al: Risk factors for worsened muscle strength after the surgical treatment of arteriovenous malformations of the eloquent motor area. $\mathbf{J}$ Neurosurg 125:289-298, 2016

16. Maldonado IL, Moritz-Gasser S, de Champfleur NM, Bertram L, Moulinié G, Duffau H: Surgery for gliomas involving the left inferior parietal lobule: new insights into the functional anatomy provided by stimulation mapping in awake patients. J Neurosurg 115:770-779, 2011

17. Parker GJ, Luzzi S, Alexander DC, Wheeler-Kingshott CA, Ciccarelli O, Lambon Ralph MA: Lateralization of ventral and dorsal auditory-language pathways in the human brain. Neuroimage 24:656-666, 2005

18. Price CJ: The anatomy of language: a review of $100 \mathrm{fMRI}$ studies published in 2009. Ann N Y Acad Sci 1191:62-88, 2010

19. Sakurai Y, Asami M, Mannen T: Alexia and agraphia with lesions of the angular and supramarginal gyri: evidence for the disruption of sequential processing. J Neurol Sci 288: $25-33,2010$

20. Southwell DG, Riva M, Jordan K, Caverzasi E, Li J, Perry DW, et al: Language outcomes after resection of dominant inferior parietal lobule gliomas. J Neurosurg 127:781-789, 2017

21. Stoeckel C, Gough PM, Watkins KE, Devlin JT: Supramarginal gyrus involvement in visual word recognition. Cortex 45:1091-1096, 2009

22. Thivard L, Hombrouck J, du Montcel ST, Delmaire C, Cohen $\mathrm{L}$, Samson S, et al: Productive and perceptive language reorganization in temporal lobe epilepsy. Neuroimage 24:841851,2005

23. Tremblay P, Dick AS: Broca and Wernicke are dead, or moving past the classic model of language neurobiology. Brain Lang 162:60-71, 2016 
24. van den Heuvel MP, Sporns O: Network hubs in the human brain. Trends Cogn Sci 17:683-696, 2013

25. Wang L, Chen D, Yang X, Olson JJ, Gopinath K, Fan T, et al: Group independent component analysis and functional MRI examination of changes in language areas associated with brain tumors at different locations. PLoS One 8:e59657, 2013

26. Xu Y, He Y, Bi Y: A tri-network model of human semantic processing. Front Psychol 8:1538, 2017

27. $\mathrm{Xu} \mathrm{Y,} \mathrm{Lin} \mathrm{Q,} \mathrm{Han} \mathrm{Z,} \mathrm{He} \mathrm{Y,} \mathrm{Bi} \mathrm{Y:} \mathrm{Intrinsic} \mathrm{functional} \mathrm{network}$ architecture of human semantic processing: Modules and hubs. Neuroimage 132:542-555, 2016

28. Yagmurlu K, Middlebrooks EH, Tanriover N, Rhoton AL Jr: Fiber tracts of the dorsal language stream in the human brain. J Neurosurg 124:1396-1405, 2016

\section{Disclosures}

The authors report no conflict of interest concerning the materials or methods used in this study or the findings specified in this paper.

\section{Author Contributions}

Conception and design: Cao, Jiao, Lin. Acquisition of data: Jiao,
Lin, $\mathrm{Wu}, \mathrm{Li}, \mathrm{Fu}, \mathrm{Huo}$. Analysis and interpretation of data: Jiao, Lin. Drafting the article: Jiao, Lin, Huo. Critically revising the article: Cao, Jiao, Lin, Li, Fu, Zhao. Reviewed submitted version of manuscript: Jiao, Lin, Wu, Li, Huo, Wang, Zhao. Statistical analysis: Jiao, Lin, Fu, Wang. Administrative/technical/material support: Wu, Wang, Zhao. Study supervision: Cao, Wang, Zhao.

\section{Supplemental Information}

Online-Only Content

Supplemental material is available with the online version of the article.

Supplemental Figs. S1-S7. https://thejns.org/doi/suppl/10.3171/ 2019.12.JNS191987.

\section{Correspondence}

Yong Cao: Beijing Tiantan Hospital, Capital Medical University, Beijing, China.caolaoban6@163.com. 